\title{
Limits on the Diffuse Gamma-Ray Background with HAWC
}

\section{Mora Durocher ${ }^{a, *}$ on behalf of the HAWC Collaboration}

(a complete list of authors can be found at the end of the proceedings)

${ }^{a}$ Physics Division, Los Alamos National Laboratory

Los Alamos, NM, USA

E-mail: mdurocher@lanl.gov

The high-energy Diffuse Gamma-Ray Background (DGRB) is expected to be produced by unresolved extra-galactic objects such as active galactic nuclei, starburst galaxies and gamma-ray bursts. At $\mathrm{TeV}$ energies, observations or stringent limits on the DGRB could have significant multi-messenger implications, such as constraining the origin of TeV-PeV astrophysical neutrinos detected by IceCube. With its continuous sensitivity to gamma rays from a few hundred $\mathrm{GeV}$ to several hundred $\mathrm{TeV}$ and its wide field-of-view, the High Altitude Water Cherenkov (HAWC) observatory is well-suited to significantly improve searches for the DGRB. In this work, strict cuts have been applied to the HAWC dataset to better isolate gamma-ray air showers from background hadronic showers. The sensitivity to the DGRB was then verified using 535 days of Crab data and Monte Carlo simulations, leading to new limits on the DGRB $>10 \mathrm{TeV}$.

$37^{\text {th }}$ International Cosmic Ray Conference (ICRC 2021)

July 12th - 23rd, 2021

Online - Berlin, Germany

\footnotetext{
${ }^{*}$ Presenter
} 


\section{Introduction}

Isotropic emission of gamma rays uncorrelated with any known sources are expected to be the main provenance of the Diffuse Gamma-Ray Background (DGRB). In 1975, the SAS-2 satellite [1] had first reported the measurement of a fainter extragalactic component of the DGRB, which was later confirmed by EGRET [2] in 1998. The DGRB being uncorrelated to any known emission source, its diffuse extragalactic component is hypothesized to originate from unresolved Active Galactic Nuclei, starburst galaxies and $\gamma$-ray bursts [3].

The High Altitude Water Cherenkov (HAWC) gamma-ray observatory is located at an altitude of 4100 meters in the state of Puebla, Mexico. It is sensitive to sources with declinations between -26 and +64 degrees, has a duty cycle of $>95 \%$ and a wide field-of-view of $\sim 2$ sr. With its 300 water Cherenkov detectors, HAWC was built to detect gamma rays in the energy range between 300 $\mathrm{GeV}$ to more than $100 \mathrm{TeV}$.

\section{Data Processing and Selection}

For ground-based detectors, such as HAWC, hadronic Cosmic Rays (CRs) are the main source of background to high-energy photon observation. Fortunately, above several TeV, the air showers produced by high-energy CRs and gamma rays differ in shape but also in composition. Two gamma/hadron separation parameters have been defined using these characteristics. Compactness [5, 6] is designed to identify muons in air showers, whereas PINCness [7] measures the smoothness of the lateral charge distribution function of air showers. By quantifying the clumpiness of air showers most of the cosmic ray contamination can further be removed.

In this work we use 535 days of data from HAWC, taken from November 2014 to June 2016. We implement a 2D binning scheme [4] focusing on high energy events with reconstructed energies above $10 \mathrm{TeV}$ (bins g to l) and where more than 61.8\% of the PMTs available were hit (bins 7 to 9). Although this analysis does not include more recent HAWC data, it was chosen for having been very well characterized when it comes to its gamma/hadron behavior with respect to the Monte Carlo simulation.

Previous studies have been performed using the HAWC observatory to set limits on the DGRB [8-10]. In this work, the DGRB region of interest is a strip centered on the Crab nebula's location. Within the strip, bright known $\gamma$-ray sources - i.e. the Crab, Geminga and the Galactic Plane have been removed to avoid contamination. The resulting DGRB strip has an area of $0.57 \mathrm{sr}$ and is shown in Figure 1.

\section{Limits on the DGRB}

\subsection{Crab Studies}

To evaluate the performance of the HAWC simulation, we compare our data to the expected $\gamma$-ray signal from the Crab nebula. The Monte Carlo simulation of gamma-rays is then made to follow the best-fit HAWC Crab spectrum [4]

$$
\left.\frac{d N}{d E}\right|_{\mathrm{Crab}}=2.35 \times 10^{-13}\left(\frac{E}{7 \mathrm{TeV}}\right)^{-2.79-0.10 \times \ln (E / 7 \mathrm{TeV})}\left(\mathrm{TeV} \mathrm{cm}^{2} \mathrm{~s}\right)^{-1}
$$




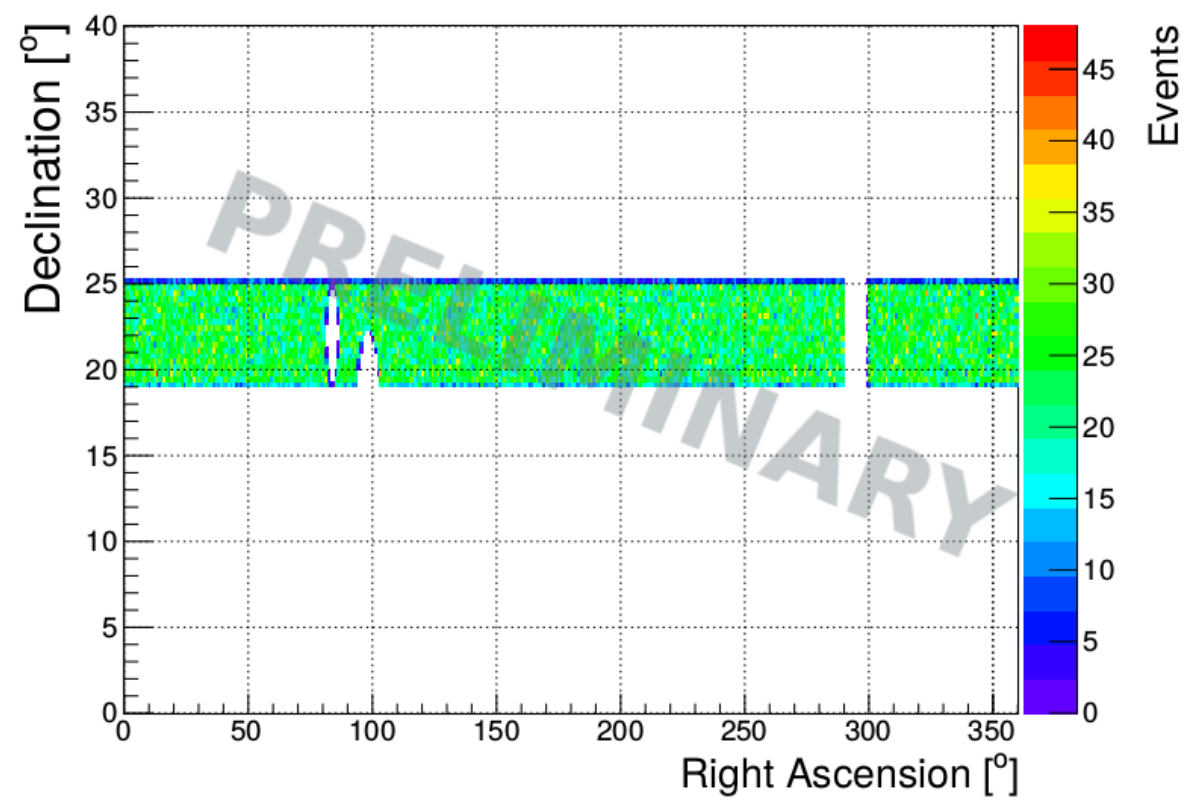

Figure 1: Map of the Diffuse Gamma-Ray Background strip

Only bins with reliable data/simulation agreement were considered, as verified using the Crab nebula.

As we apply tighter gamma/hadron separation cuts, few events remain and we must rely on Poisson statistics. For a binned likelihood analysis, the log-likelihood is calculated as the sum of the log of the Poisson probability to observe $N^{o b s}$ events in a bin given that the model predicts $N^{\text {pred }}$

$$
\ln \mathcal{L}=\sum_{i}^{\text {bins }} N_{i}^{\text {obs }} \log \left(N_{i}^{\text {pred }}\right)-\sum_{i}^{\text {bins }} N_{i}^{\text {pred }}
$$

where $N^{o b s}$ is set as the number of events in our DGRB strip and $N^{\text {pred }}$ depends on the number of simulated Crab events. However we do not perform a joint likelihood; in order to calculate the best estimate for the overall scale of the spectrum, the bins are not summed but treated as separate independent "experiments". The $95 \%$ one-sided upper limit $-2 \Delta \ln \mathcal{L}=2.71$ - is calculated in each bin and the one with the lowest value is selected, as it would be the one with the most expansive limit.

By spreading the HAWC Crab spectrum over the 0.57 sr solid angle of the DGRB strip, we can then calculate the $95 \%$ containment level of the best estimate for the overall scale of the spectrum, referred to as $\beta_{95 \%}$

$$
\left.\frac{d^{2} N}{d E d \Omega}\right|_{95 \%}=\beta_{95 \%} \times\left.\frac{d N}{d E}\right|_{\text {Crab }}
$$

For the energy bins studied, chosing the $\beta_{95 \%}$ with the lowest value will lead to their corresponding fraction hit bin, the results of which can be found in Table 1. Furthermore, Figure 2 shows the upper-limits for each energy bin compared to other observations and limits. 


\begin{tabular}{cccc}
\hline Energy bin & Fraction hit bin & $\beta_{95 \%}$ & Simulation median energy (TeV) \\
\hline $\mathrm{g}$ & 7 & 308 & 19 \\
$\mathrm{~h}$ & 8 & 192 & 23 \\
$\mathrm{i}$ & 9 & 145 & 40 \\
$\mathrm{j}$ & 9 & 57.8 & 69 \\
$\mathrm{k}$ & 9 & 37.1 & 106 \\
$\mathrm{l}$ & 9 & 61.0 & 168 \\
\hline
\end{tabular}

Table 1: The results of the binned maximum likelihood analysis. For each energy bin and its corresponding fraction hit bin, the column labeled "Simulation median energy" displays the median energy from simulation assuming the HAWC Crab log parabola spectrum.

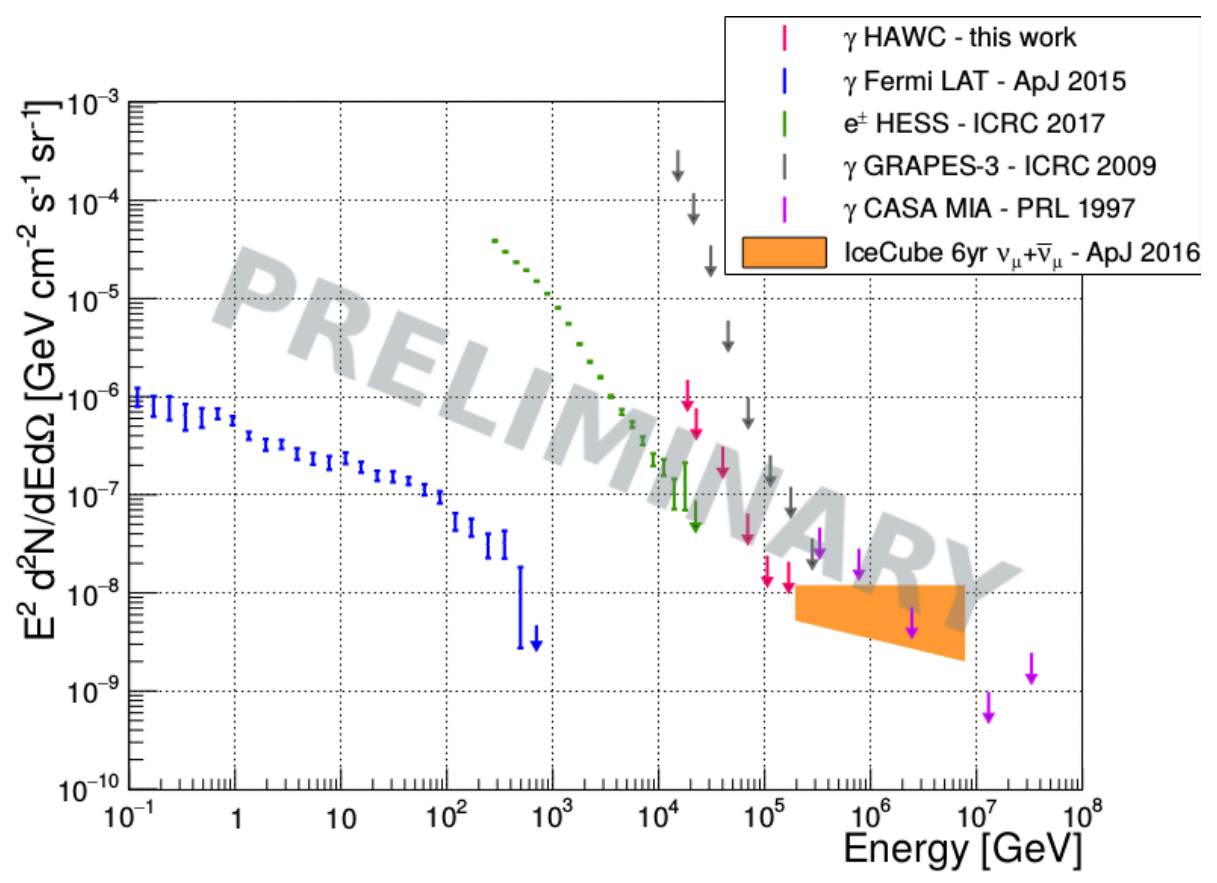

Figure 2: Limits on the DGRB using 535 days of HAWC data compared to the diffuse electron/positron flux observed by HESS [11, 12]. Also shown is the observed IGRB by the Fermi-LAT [13], the gamma-ray flux corresponding to the IceCube $v_{\mu}+\bar{v}_{\mu}$ astrophysical flux [14], as well as previous high-energy limits by GRAPES [15] and CASA-MIA [16].

\subsection{Model Testing}

Now we evaluate the data in the DGRB strip with respect to the spectra from other models. We then employ the same bins and apply the same cuts as those obtained using the HAWC Crab spectrum, from which we choose the most expansive limit overall.

The gamma-ray production is predicted to have a similar spectrum and flux as the neutrinos seen by IceCube. We made use of a relation between the fluxes of gamma rays and neutrinos from [17] to find the gamma-ray flux corresponding to the best fit with the IceCube unbroken power-law 
model for the $v_{\mu}+\bar{v}_{\mu}$ astrophysical flux [14]

$$
\left.\frac{d^{2} N}{d E d \Omega}\right|_{\mathrm{IC}}=9.0 \times 10^{-16}\left(\frac{E}{100 \mathrm{TeV}}\right)^{-2.13}\left(\mathrm{TeV} \mathrm{cm}^{2} \mathrm{~s} \mathrm{sr}\right)^{-1}
$$

The HESS observatory has detected an isotropic CR flux of electrons and positrons up to 20 $\mathrm{TeV}[11,12]$. Although the limits obtained in this work are pushing into an energy range higher than the H.E.S.S. observations, we can still make a comparison with the best fit for the H.E.S.S. electron/positron flux with a smooth broken power law

$$
\left.\frac{d^{2} N}{d E d \Omega}\right|_{\text {H.E.S.S. }}=1.05 \times 10^{-8}\left(\frac{E}{1 \mathrm{TeV}}\right)^{-3.04}\left(1+\left(\frac{E}{0.94 \mathrm{TeV}}\right)^{\frac{1}{0.12}}\right)^{0.12 \times(3.04-3.78)}\left(\mathrm{TeV} \mathrm{cm}^{2} \mathrm{~s} \mathrm{sr}\right)^{-1}
$$

The resulting $\beta_{95 \%}$ and $\beta_{\text {best }}$ from injecting the previous spectra are shown in Table 2.

\begin{tabular}{lcc}
\hline & $\beta_{95 \%}$ & $\beta_{\text {best }}$ \\
\hline IceCube spectrum & 2.13 & 1.32 \\
H.E.S.S spectrum & 9.60 & 6.94 \\
\hline
\end{tabular}

Table 2: The results for the model testing. $\beta_{\text {best }}$ refers to the best estimate of the overall scale of the spectrum - for which $-2 \ln \mathcal{L}$ is at its minimum - while $\beta_{95 \%}$ corresponds to its $95 \%$ containment level.

\section{Discussion and Prospects}

In addition to DGRB events, the flux observed by HAWC may also include other isotropic events that would be considered as background. One possible component of this flux would be misreconstructed hadrons, due to their gamma-like appearance in the HAWC detector. Another possible source of isotropic emission would be CR electrons and positrons whose air showers are similar to those induced by gamma rays. A better understanding of the expected CR contamination would help break the degeneracy.

Astrophysical pion production emit neutrinos in addition to $\gamma$-rays, and so might dark matter annihilations. Interestingly, the best estimate of the overall scale of the spectrum is only 1.32 times the IceCube flux. This value is likely to include a fair amount of CR background and should definitely not be considered as a measurement of the DGRB. Nonetheless, despite being ambiguous, this value is fairly consistent with the IceCube flux itself. Better characterization of residual CRs is underway and could verify the consistency between gamma-ray and neutrino flux.

With the addition of more years of data, upcoming improvements to the HAWC reconstruction algorithms and analytical methods, and the deployment of the outrigger array in 2018, stronger constraints on the previous results are expected. Furthermore, a next-generation Southern Widefield Gamma-ray Observatory (SWGO) ${ }^{1}$ is being considered for the Southern Hemisphere which will extend sensitivity to energies above the tens of PeV.

\footnotetext{
${ }^{1}$ Www.swgo.org
} 


\section{Acknowledgments}

We acknowledge the support from: the US National Science Foundation (NSF); the US Department of Energy Office of High-Energy Physics; the Laboratory Directed Research and Development (LDRD) program of Los Alamos National Laboratory; Consejo Nacional de Ciencia y Tecnología (CONACyT), México, grants 271051, 232656, 260378, 179588, 254964, 258865, 243290, 132197, A1-S-46288, A1-S-22784, cátedras 873, 1563, 341, 323, Red HAWC, México; DGAPA-UNAM grants IG101320, IN111716-3, IN111419, IA102019, IN110621, IN110521; VIEP-BUAP; PIFI 2012, 2013, PROFOCIE 2014, 2015; the University of Wisconsin Alumni Research Foundation; the Institute of Geophysics, Planetary Physics, and Signatures at Los Alamos National Laboratory; Polish Science Centre grant, DEC-2017/27/B/ST9/02272; Coordinación de la Investigación Científica de la Universidad Michoacana; Royal Society - Newton Advanced Fellowship 180385; Generalitat Valenciana, grant CIDEGENT/2018/034; Chulalongkorn University's CUniverse (CUAASC) grant; Coordinación General Académica e Innovación (CGAI-UdeG), PRODEP-SEP UDG-CA499; Institute of Cosmic Ray Research (ICRR), University of Tokyo, H.F. acknowledges support by NASA under award number 80GSFC21M0002. We also acknowledge the significant contributions over many years of Stefan Westerhoff, Gaurang Yodh and Arnulfo Zepeda Dominguez, all deceased members of the HAWC collaboration. Thanks to Scott Delay, Luciano Díaz and Eduardo Murrieta for technical support.

\section{References}

[1] C.E. Fichtel, R.C. Hartman, D.A. Kniffen, D.J. Thompson, G.F. Bignami, H. Ögelman et al., High-energy gamma-ray results from the second Small Astronomy Satellite, Astrophys. J. 198 (1975) 163.

[2] EGRET collaboration, EGRET observations of the extragalactic gamma-ray emission, Astrophys. J. 494 (1998) 523 [astro-ph/9709257].

[3] C.D. Dermer, The Extragalactic Gamma Ray Background, AIP Conf. Proc. 921 (2007) 122 [0704.2888].

[4] A.U. Abeysekara, A. Albert, R. Alfaro, C. Alvarez, J.D. Álvarez, J.R.A. Camacho et al., Measurement of the Crab Nebula Spectrum Past $100 \mathrm{TeV}$ with HAWC, The Astrophysical Journal 881 (2019) 134.

[5] A. Abeysekara et al., On the sensitivity of the HAWC observatory to gamma-ray bursts, Astroparticle Physics 35 (2012) 641 .

[6] A. Abeysekara et al., Sensitivity of the High Altitude Water Cherenkov Detector to Sources of Multi-TeV Gamma Rays, Astropart. Phys. 50-52 (2013) 26 [1306 . 5800].

[7] A. Abeysekara et al., Observation of the Crab Nebula with the HAWC Gamma-Ray Observatory, Astrophys. J. 843 (2017) 39 [1701.01778].

[8] J. Pretz, Limit on an Isotropic Diffuse Gamma-Ray Population with HAWC, 2015. 
[9] H. Zhou, C.D. Rho and G. Vianello, Probing Galactic Diffuse TeV Gamma-Ray Emission with the HAWC Observatory, 2017.

[10] J.P. Harding, Constraints on the Diffuse Gamma-Ray Background with HAWC, 2019.

[11] D. Kerszberg et al., The cosmic-ray electron spectrum measured with H.E.S.S., .

[12] H.E.S.S. Collaboration, Probing Local Sources with High Energy Cosmic Ray Electrons, .

[13] M. Ackermann et al., The spectrum of isotropic diffuse gamma-ray emission between 100 MeV and $820 \mathrm{GeV}$, Astrophys. J. 799 (2015) 86.

[14] ICECubE collaboration, Observation and Characterization of a Cosmic Muon Neutrino Flux from the Northern Hemisphere using six years of IceCube data, Astrophys. J. 833 (2016) 3 [1607.08006].

[15] M. Minamino et al., Upper Limit on the Diffuse Gamma Ray Flux using GRAPES-3 Experiment, .

[16] M.C. Chantell et al., Limits on the Isotropic Diffuse Flux of Ultrahigh Energy Gamma Radiation, Phys. Rev. Lett. 79 (1997) 1805.

[17] H.A.A. Solares, S. Coutu, J.J. DeLaunay, D.B. Fox, T. Grégoire, A. Keivani et al., Multimessenger Gamma-Ray and Neutrino Coincidence Alerts Using HAWC and IceCube Subthreshold Data, The Astrophysical Journal 906 (2021) 63. 


\section{Full Authors List: HAWC Collaboration}

A.U. Abeysekara ${ }^{48}$, A. Albert ${ }^{21}$, R. Alfaro ${ }^{14}$, C. Alvarez ${ }^{41}$, J.D. Álvarez ${ }^{40}$, J.R. Angeles Camacho ${ }^{14}$, J.C. Arteaga-Velázquez ${ }^{40}$, K. P. Arunbabu $^{17}$, D. Avila Rojas ${ }^{14}$, H.A. Ayala Solares ${ }^{28}$, R. Babu ${ }^{25}$, V. Baghmanyan ${ }^{15}$, A.S. Barber ${ }^{48}$, J. Becerra Gonzalez ${ }^{11}$, E. BelmontMoreno $^{14}$, S.Y. BenZvi ${ }^{29}$, D. Berley ${ }^{39}$, C. Brisbois ${ }^{39}$, K.S. Caballero-Mora ${ }^{41}$, T. Capistrán ${ }^{12}$, A. Carramiñana ${ }^{18}$, S. Casanova ${ }^{15}$, O. Chaparro-Amaro $^{3}$, U. Cotti ${ }^{40}$, J. Cotzomi $^{8}$, S. Coutiño de León ${ }^{18}$, E. De la Fuente ${ }^{46}$, C. de León ${ }^{40}$, L. Diaz-Cruz ${ }^{8}$, R. Diaz Hernandez ${ }^{18}$, J.C. Díaz-Vélez ${ }^{46}$, B.L. Dingus ${ }^{21}$, M. Durocher ${ }^{21}$, M.A. DuVernois ${ }^{45}$, R.W. Ellsworth ${ }^{39}$, K. Engel ${ }^{39}$, C. Espinoza ${ }^{14}$, K.L. Fan ${ }^{39}$, K. Fang $^{45}$, M. Fernández Alonso ${ }^{28}$, B. Fick ${ }^{25}$, H. Fleischhack ${ }^{51,11,52}$, J.L. Flores ${ }^{46}$, N.I. Fraija ${ }^{12}$, D. Garcia ${ }^{4}$, J.A. García-González ${ }^{20}$, J. L. García-Luna ${ }^{46}$, G. García-Torales ${ }^{46}$, F. Garfias ${ }^{12}$, G. Giacinti ${ }^{22}$, H. Goksu ${ }^{22}$, M.M. González ${ }^{12}$, J.A. Goodman ${ }^{39}$, J.P. Harding ${ }^{21}$, S. Hernandez $^{14}$, I. Herzog ${ }^{25}$, J. Hinton ${ }^{22}$, B. Hona ${ }^{48}$, D. Huang ${ }^{25}$, F. Hueyotl-Zahuantitla ${ }^{41}$, C.M. Hui ${ }^{23}$, B. Humensky ${ }^{39}$, P. Hüntemeyer ${ }^{25}$, A. Iriarte ${ }^{12}$, A. Jardin-Blicq ${ }^{22,49,50}$, H. Jhee ${ }^{43}$, V. Joshi ${ }^{7}$, D. Kieda ${ }^{48}$, G J. Kunde ${ }^{21}$, S. Kunwar ${ }^{22}$, A. Lara ${ }^{17}$, J. Lee ${ }^{43}$, W.H. Lee ${ }^{12}$, D. Lennarz ${ }^{9}$, H. León Vargas ${ }^{14}$, J. Linnemann ${ }^{24}$, A.L. Longinotti ${ }^{12}$, R. López-Coto ${ }^{19}$, G. Luis-Raya ${ }^{44}$, J. Lundeen ${ }^{24}$, K. Malone ${ }^{21}$, V. Marandon $^{22}$, O. Martinez ${ }^{8}$, I. Martinez-Castellanos ${ }^{39}$, H. Martínez-Huerta ${ }^{38}$, J. Martínez-Castro ${ }^{3}$, J.A.J. Matthews ${ }^{42}$, J. McEnery ${ }^{11}$, P. Miranda-Romagnoli $^{34}$, J.A. Morales-Soto ${ }^{40}$, E. Moreno ${ }^{8}$, M. Mostafá ${ }^{28}$, A. Nayerhoda ${ }^{15}$, L. Nellen ${ }^{13}$, M. Newbold ${ }^{48}$, M.U. Nisa ${ }^{24}$, R. Noriega-Papaqui ${ }^{34}$, L. Olivera-Nieto ${ }^{22}$, N. Omodei ${ }^{32}$, A. Peisker ${ }^{24}$, Y. Pérez Araujo ${ }^{12}$, E.G. Pérez-Pérez ${ }^{44}$, C.D. Rho ${ }^{43}$, C. Rivière ${ }^{39}$, D. Rosa-Gonzalez $^{18}$, E. Ruiz-Velasco ${ }^{22}$, J. Ryan ${ }^{26}$, H. Salazar ${ }^{8}$, F. Salesa Greus ${ }^{15,53}$, A. Sandoval ${ }^{14}$, M. Schneider ${ }^{39}$, H. Schoorlemmer ${ }^{22}$, J. Serna-Franco ${ }^{14}$, G. Sinnis ${ }^{21}$, A.J. Smith ${ }^{39}$, R.W. Springer ${ }^{48}$, P. Surajbali ${ }^{22}$, I. Taboada ${ }^{9}$, M. Tanner ${ }^{28}$, K. Tollefson ${ }^{24}$, I. Torres ${ }^{18}$, R. Torres-Escobedo $^{30}$, R. Turner ${ }^{25}$, F. Ureña-Mena ${ }^{18}$, L. Villaseñor ${ }^{8}$, X. Wang ${ }^{25}$, I.J. Watson ${ }^{43}$, T. Weisgarber ${ }^{45}$, F. Werner ${ }^{22}$, E. Willox ${ }^{39}$, J. Wood ${ }^{23}$, G.B. Yodh ${ }^{35}$, A. Zepeda ${ }^{4}$, H. Zhou ${ }^{30}$

${ }^{1}$ Barnard College, New York, NY, USA, ${ }^{2}$ Department of Chemistry and Physics, California University of Pennsylvania, California, PA, USA, ${ }^{3}$ Centro de Investigación en Computación, Instituto Politécnico Nacional, Ciudad de México, México, ${ }^{4}$ Physics Department, Centro de Investigación y de Estudios Avanzados del IPN, Ciudad de México, México, ${ }^{5}$ Colorado State University, Physics Dept., Fort Collins, CO, USA, ${ }^{6}$ DCI-UDG, Leon, Gto, México, ${ }^{7}$ Erlangen Centre for Astroparticle Physics, Friedrich Alexander Universität, Erlangen, BY, Germany, ${ }^{8}$ Facultad de Ciencias Físico Matemáticas, Benemérita Universidad Autónoma de Puebla, Puebla, México, ${ }^{9}$ School of Physics and Center for Relativistic Astrophysics, Georgia Institute of Technology, Atlanta, GA, USA, ${ }^{10}$ School of Physics Astronomy and Computational Sciences, George Mason University, Fairfax, VA, USA, ${ }^{11}$ NASA Goddard Space Flight Center, Greenbelt, MD, USA, ${ }^{12}$ Instituto de Astronomía, Universidad Nacional Autónoma de México, Ciudad de México, México, ${ }^{13}$ Instituto de Ciencias Nucleares, Universidad Nacional Autónoma de México, Ciudad de México, México, ${ }^{14}$ Instituto de Física, Universidad Nacional Autónoma de México, Ciudad de México, México, ${ }^{15}$ Institute of Nuclear Physics, Polish Academy of Sciences, Krakow, Poland, ${ }^{16}$ Instituto de Física de São Carlos, Universidade de São Paulo, São Carlos, SP, Brasil, ${ }^{17}$ Instituto de Geofísica, Universidad Nacional Autónoma de México, Ciudad de México, México, ${ }^{18}$ Instituto Nacional de Astrofísica, Óptica y Electrónica, Tonantzintla, Puebla, México, ${ }^{19}$ INFN Padova, Padova, Italy, ${ }^{20}$ Tecnologico de Monterrey, Escuela de Ingeniería y Ciencias, Ave. Eugenio Garza Sada 2501, Monterrey, N.L., 64849, México, ${ }^{21}$ Physics Division, Los Alamos National Laboratory, Los Alamos, NM, USA, ${ }^{22}$ Max-Planck Institute for Nuclear Physics, Heidelberg, Germany, ${ }^{23}$ NASA Marshall Space Flight Center, Astrophysics Office, Huntsville, AL, USA, ${ }^{24}$ Department of Physics and Astronomy, Michigan State University, East Lansing, MI, USA, ${ }^{25}$ Department of Physics, Michigan Technological University, Houghton, MI, USA, ${ }^{26}$ Space Science Center, University of New Hampshire, Durham, NH, USA, ${ }^{27}$ The Ohio State University at Lima, Lima, OH, USA, ${ }^{28}$ Department of Physics, Pennsylvania State University, University Park, PA, USA, ${ }^{29}$ Department of Physics and Astronomy, University of Rochester, Rochester, NY, USA, ${ }^{30}$ Tsung-Dao Lee Institute and School of Physics and Astronomy, Shanghai Jiao Tong University, Shanghai, China, ${ }^{31}$ Sungkyunkwan University, Gyeonggi, Rep. of Korea, ${ }^{32}$ Stanford University, Stanford, CA, USA, ${ }^{33}$ Department of Physics and Astronomy, University of Alabama, Tuscaloosa, AL, USA, ${ }^{34}$ Universidad Autónoma del Estado de Hidalgo, Pachuca, Hgo., México, ${ }^{35}$ Department of Physics and Astronomy, University of California, Irvine, Irvine, CA, USA, ${ }^{36}$ Santa Cruz Institute for Particle Physics, University of California, Santa Cruz, Santa Cruz, CA, USA, ${ }^{37}$ Universidad de Costa Rica, San José, Costa Rica, ${ }^{38}$ Department of Physics and Mathematics, Universidad de Monterrey, San Pedro Garza García, N.L., México, ${ }^{39}$ Department of Physics, University of Maryland, College Park, MD, USA, ${ }^{40}$ Instituto de Física y Matemáticas, Universidad Michoacana de San Nicolás de Hidalgo, Morelia, Michoacán, México, ${ }^{41}$ FCFM-MCTP, Universidad Autónoma de Chiapas, Tuxtla Gutiérrez, Chiapas, México, ${ }^{42}$ Department of Physics and Astronomy, University of New Mexico, Albuquerque, NM, USA, ${ }^{43}$ University of Seoul, Seoul, Rep. of Korea, ${ }^{44}$ Universidad Politécnica de Pachuca, Pachuca, Hgo, México, ${ }^{45}$ Department of Physics, University of Wisconsin-Madison, Madison, WI, USA, ${ }^{46}$ CUCEI, CUCEA, Universidad de Guadalajara, Guadalajara, Jalisco, México, ${ }^{47}$ Universität Würzburg, Institute for Theoretical Physics and Astrophysics, Würzburg, Germany, ${ }^{48}$ Department of Physics and Astronomy, University of Utah, Salt Lake City, UT, USA, ${ }^{49}$ Department of Physics, Faculty of Science, Chulalongkorn University, Pathumwan, Bangkok 10330, Thailand, ${ }^{50}$ National Astronomical Research Institute of Thailand (Public Organization), Don Kaeo, MaeRim, Chiang Mai 50180, Thailand, ${ }^{51}$ Department of Physics, Catholic University of America, Washington, DC, USA, ${ }^{52}$ Center for Research and Exploration in Space Science and Technology, NASA/GSFC, Greenbelt, MD, USA, ${ }^{53}$ Instituto de Física Corpuscular, CSIC, Universitat de València, Paterna, Valencia, Spain 